Revista Perspectivas Online: Exatas \& Engenharias Janeiro de 2018, Vol.8, no 20, p. 13-24

ISSN: $2236-885 \mathrm{X}$ (Online)

DOI: $10.25242 / 885 \times 82020181300$

\title{
CONFIABILIDADE DE ESTACAS EMPREGANDO MÉTODO DE ESTIMATIVAS PONTUAIS
}

\author{
Aline de Viegas Beloni ${ }^{1}$, Bruna Sá Britto Valério ${ }^{1}$, Antônio Marcos de Lima Alves ${ }^{1}$ \\ \& Mauro de Vasconcellos Real ${ }^{*}$
}

\begin{abstract}
RESUMO
BELONI, A. V.; VALÉRIO, B.S.B.; ALVES, A.M.L.; REAL, M.V. Confiabilidade de estacas empegando método de estimativas pontuais. Perspectivas Online: Exatas \& Engenharias, v. 8, n.20, p.13-24,2018.
\end{abstract}

O objetivo do presente trabalho é a análise de confiabilidade quanto à ruptura geotécnica das estacas de uma área do cais modernizado do Porto Novo de Rio Grande, RS, Brasil. O carregamento característico sobre as estacas foi obtido a partir de dados descritos no memorial de cálculo da obra, considerando-se diversas relações entre as parcelas permanente e variável das cargas. A capacidade de carga das estacas foi estimada a partir das negas medidas ao final da cravação, através da adoção de uma fórmula dinâmica. O Método das Estimativas Pontuais foi adotado na avaliação das dispersões dos carregamentos e das capacidades de carga das estacas. A partir das curvas de distribuição de probabilidades dos carregamentos e das capacidades de carga, foram calculados os índices de confiabilidade e as probabilidades de ruína da área do cais em estudo, para diversos cenários de variabilidade dos carregamentos. De acordo com a literatura na área da engenharia de fundações, são geralmente considerados aceitáveis valores de índices de confiabilidade superiores a 3,09, que conduzem a probabilidades de ruína inferiores a 1/1000 eventos. Os resultados obtidos neste estudo apresentam-se superiores ao valor indicado na literatura, portanto comprovando a segurança e a confiabilidade da obra.

Palavras-chave: Fundações; Confiabilidade; Método das Estimativas Pontuais.

Persp. Online: exatas \& eng., Campos dos Goytacazes, 20 (08) 13-24 - 2018 


\begin{abstract}
The objective of the present work is the reliability analysis regarding the geotechnical rupture of the piles of an area of the modernized dock of Porto Novo of Rio Grande, RS, Brazil. The characteristic load on the piles was obtained from data described in the calculation memorial, considering several relationships between the permanent and variable parcels of the loads. The load capacity of the piles was estimated from the sets measured at the end of the driving, through the adoption of a dynamic formula. The Point Estimate Method was adopted in the

the bearing capacities of the piles. From the probability distribution curves of the loads and the bearing capacities, the reliability indexes and the probabilities of ruin of the area of the quay under study were calculated for several scenarios of load variability. According to the technical literature, reliability index values greater than 3.09 are generally acceptable, leading to ruin probabilities of less than 1/1000 events. The results obtained in this study are higher than the value indicated in the literature, thus proving the safety and reliability of the job.
\end{abstract} evaluation of the dispersions of the loads and

Keywords: Foundations; Reliability; Point Estimate Method.

\footnotetext{
${ }^{1}$ Universidade Federal do Rio Grande, FURG - Rua Visc. De Paranaguá, 102, Centro, Rio Grande, RS, CEP: $96203-$ 900, Brasil;

(*)e-mail: mauroreal@furg.com

Data de chegada: Aceito para publicação:
} 


\section{INTRODUÇÃO}

Para determinação do nível de confiabilidade de um determinado processo ou sistema, é necessária uma avaliação cuidadosa das incertezas associadas à sua capacidade e à sua demanda. Essa avaliação, em geral, pode ser realizada convenientemente aplicando-se ferramentas probabilísticas, que permitem estudar os efeitos combinados das incertezas, incorporando-os na análise.

Em especial, o projeto de fundações de qualquer tipo de estrutura envolve incertezas oriundas de várias fontes (AOKI, 2002). Algumas destas fontes são: variabilidade espacial das propriedades do solo; investigação geotécnica limitada; alterações no comportamento do solo ocorridas durante e após a execução da fundação; simplificações adotadas nos modelos de cálculo; desconhecimento do exato mecanismo de interação solo-fundação; variabilidade na magnitude das cargas aplicadas aos elementos de fundação.

Lacasse e Nadim (1998) afirmam que a estatística, as análises de confiabilidade e as estimativas de risco podem ser ferramentas de apoio às decisões muito úteis na Engenharia Geotécnica, e avaliam as razões pelas quais este tipo de estudo é raro na prática. Segundo Duncan (2000), as teorias probabilísticas de análise de confiabilidade podem ser aplicadas na Engenharia Geotécnica através de procedimentos simples, sem exigir mais dados além daqueles normalmente necessários para uma análise determinística convencional. Com um esforço adicional relativamente pequeno, é possível um refinamento considerável na avaliação da segurança e confiabilidade de um projeto geotécnico. De Mello (2002) também defende a aplicação de análises probabilísticas simples aos projetos geotécnicos, visando rechaçar o que o autor chama de "receitasdogmas" em prol da incorporação de dados experimentais à avaliação de incertezas.

Destaca-se que os fatores de segurança exigidos pelas normas não asseguram a ausência de risco de ruína. Neste contexto, considera-se que não existe projeto cem por cento seguro. Portanto, para projetos geotécnicos de fundações, além de garantir os fatores de segurança propostos por norma, deve-se estimar uma determinada probabilidade de ruína aceitável para a fundação.

Neste sentido, esse estudo tem como objetivo determinar o índice de confiabilidade e a probabilidade de ruína de uma área do cais modernizado do Porto Novo de Rio Grande (RS), a partir do Método Probabilístico de Estimativas Pontuais, também conhecido como Método de Rosenblueth.

\section{DESCRIÇÃO DA OBRA}

A obra descrita neste estudo é a modernização do Cais do Porto Novo, localizado na cidade de Rio Grande (RS). O cais original do Porto Novo, composto de um muro formado por seis blocos de concreto sobrepostos, foi inaugurado em 1915, tendo sido projetado para receber navios com calado de até $10 \mathrm{~m}$. Recentemente, o cais tem recebido reformas e ampliações, visando principalmente o aprofundamento do calado para $14 \mathrm{~m}$, possibilitando assim a atracação de navios de maior porte.

A primeira etapa da obra de modernização envolveu a construção de um novo cais com $450 \mathrm{~m}$ de extensão, apoiado no antigo muro de cais pelo lado de terra, e em 72 novas estacas mistas pelo lado de mar. O projeto foi dividido em seis módulos, cada um com $75 \mathrm{~m}$ de extensão, constando de uma plataforma rígida em seu plano, três vigas paralelas ao cais, estacas Franki (terra) e estacas mistas (mar), conforme exposto na Figura 1. As forças perpendiculares ao cais, no sentido terra-mar, são absorvidas por grupos de tirantes que partem dos blocos e são ancorados no solo e as forças paralelas ao cais são absorvidas pelo atrito do solo com o paramento posterior. 


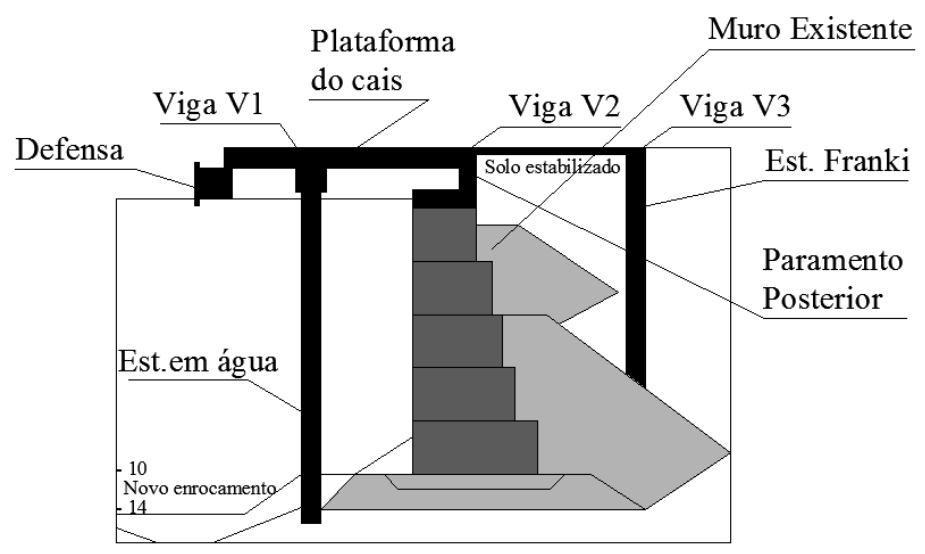

Figura 1: Seção transversal do cais (Lobo, 2003)

Para o projeto, foi realizada uma campanha de ensaios do tipo SPT ("Standard Penetration Test"), composta de furos de sondagem a cada $100 \mathrm{~m}$, tanto do lado do mar quanto em terra firme. Foram executados 12 furos de sondagem, sendo que destes, 7 foram executados sob lâmina d'água (S1A, S2B, S3B, S4A, S5, S6 e S7) e 5 em terra firme (S8A,S9B, S10, S11 e S12C). Os boletins de sondagens podem ser encontrados em Lobo (2003) e Magalhães (2011). Na Figura 2 encontra-se um perfil geotécnico esquemático, baseado nos resultados das sondagens de mar.

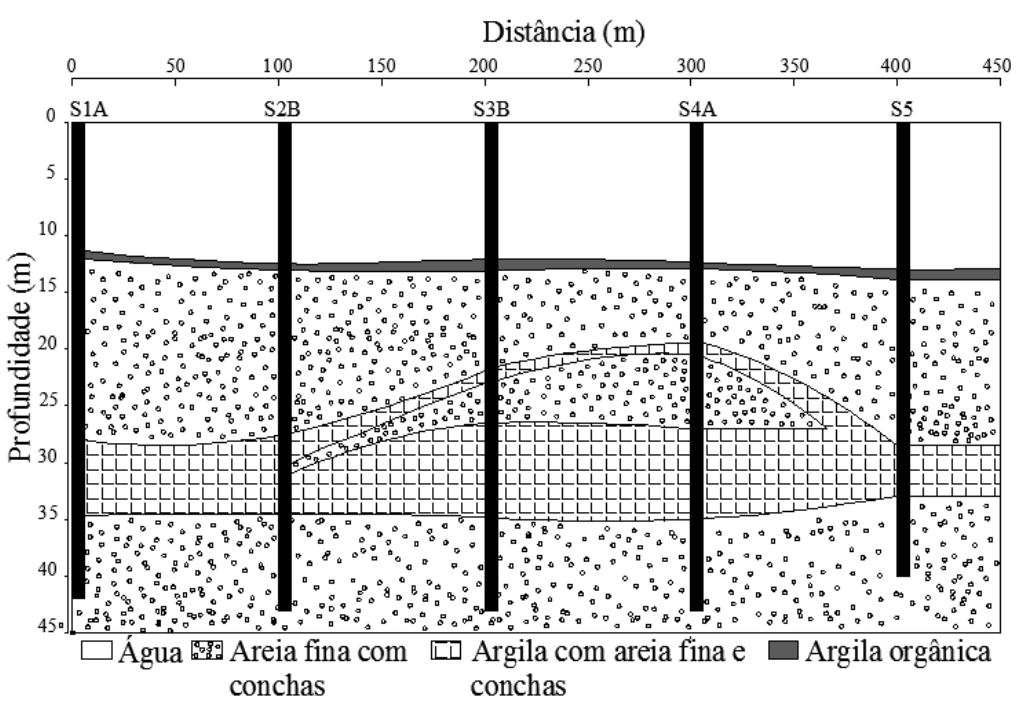

Figura 2: Perfil longitudinal em água

As estacas de mar são mistas (tubos metálicos preenchidos parcialmente com concreto armado). $\mathrm{O}$ tubo metálico tem comprimento total de $48 \mathrm{~m}$, diâmetro externo de $0,7112 \mathrm{~m}$ (28") e espessura de 9,53 mm. O tubo é preenchido com concreto armado da cota $+1,05 \mathrm{~m}$ à cota $-21,00 \mathrm{~m}$. O aço que constitui os tubos metálicos das estacas possui uma tensão de escoamento de $316 \mathrm{MPa}$ e o módulo de elasticidade é de 210 $\mathrm{GPa}$. O concreto usado para o preenchimento das estacas possui resistência característica fck $\geq 30 \mathrm{MPa}$ e aço CA-50, com cobrimento de $5 \mathrm{~cm}$.

De acordo com o projeto, cada estaca recebe em seu topo uma carga máxima de compressão de 3000 $\mathrm{kN}$ e um momento fletor de $310 \mathrm{kNm}$ em qualquer direção. A cravação das estacas foi realizada com o martelo modelo DELMAG D-44 (martelo automático a diesel).

Durante a execução do estaqueamento, foi anotado o boletim de cravação de todas as estacas, bem como efetuada a medida de nega ao final da cravação. Também foram realizados ensaios de carregamento dinâmico, em um número mínimo de estacas como preconizado pela NBR 6122 (2010). 


\section{MÉTODO PROBABILÍSTICO DE ESTIMATIVAS PONTUAIS}

O método das Estimativas Pontuais, proposto por Rosenblueth (1975) e estendido por Rosenblueth (1981), permite que, conhecendo-se a variabilidade de diversas variáveis aleatórias independentes, estime-se a variabilidade de uma variável aleatória dependente daquelas (ALVES E SANTA MARIA, 2001).

Segundo Rosenblueth (1975), dada uma função real $Y$ de uma variável aleatória real $X$, e os primeiros dois ou três momentos probabilísticos de $X$, expressões podem ser desenvolvidas para os momentos probabilísticos de $Y$, como combinações lineares de potências de estimativas pontuais $y\left(x_{+}\right)$e $y\left(x_{\text {- }}\right.$ ), onde $x_{+}$e $x_{-}$são valores específicos de $X$. As aproximações podem ser generalizadas para o caso em que $Y$ é função de várias variáveis aleatórias.

De acordo com o método, para uma função com duas variáveis aleatórias $Y=f\left(X_{1}, X_{2}\right)$, tem-se que o valor esperado de $Y$ será:

$$
E[Y]=p_{y++} y_{++}+p_{y+-} y_{+-}+p_{y-+} y_{-+}+p_{y--} y_{--}
$$

Onde:

$$
\begin{aligned}
& p_{y++}=p_{y--}=\frac{1}{2^{2}}\left(1+\rho_{12}\right) \\
& p_{y+-}=p_{y-+}=\frac{1}{2^{2}}\left(1-\rho_{12}\right) \\
& y_{ \pm \pm}=\left(m_{x_{1}} \pm \sigma_{x_{1}}, m_{x_{2}} \pm \sigma_{x_{2}}\right)
\end{aligned}
$$

Para uma função com três variáveis aleatórias $Y=f\left(X_{1}, X_{2}, X_{3}\right)$, o valor esperado de $Y$ será:

$$
\begin{aligned}
& E[Y]=p_{y+++} y_{+++}+p_{y---} y_{---}+p_{y++-} y_{++-}+p_{y-+} y_{--+}+p_{y+-+} y_{+-+}+p_{y-+-} y_{-+-} \\
& +p_{y+--} y_{+--}+p_{y-+} y_{-++}
\end{aligned}
$$

Sendo:

$$
\begin{aligned}
& p_{y+++}=p_{y---}=\frac{1}{2^{3}}\left(1+\rho_{12}+\rho_{23}+\rho_{31}\right) \\
& p_{y++-}=p_{y-+}=\frac{1}{2^{3}}\left(1+\rho_{12}-\rho_{23}-\rho_{31}\right) \\
& p_{y+-+}=p_{y-+-}=\frac{1}{2^{3}}\left(1-\rho_{12}-\rho_{23}+\rho_{31}\right) \\
& p_{y+--}=p_{y-++}=\frac{1}{2^{3}}\left(1-\rho_{12}+\rho_{23}-\rho_{31}\right)
\end{aligned}
$$




$$
y_{ \pm \pm \pm}=Y\left(m_{x_{1}} \pm \sigma_{x_{1}}, m_{x_{2}} \pm \sigma_{x_{2}}, m_{x_{3}} \pm \sigma_{x_{3}}\right)
$$

onde $\rho_{i j}$ são os valores dos coeficientes de correlação entre as duas variáveis aleatórias conhecidas $X_{i}$ e $X_{j}$, e $m_{x i}$ e $\sigma_{x i}$ são respectivamente a média e o desvio padrão da distribuição da variável aleatória conhecida $X_{i}$.

O desvio padrão de $Y$ é dado pela seguinte equação:

$$
\sigma_{y}=\sqrt{E\left[y^{2}\right]-(E[y])^{2}}
$$

\section{ANÁLISE PROBABILÍSTICA DA CAPACIDADE DE CARGAS DAS ESTACAS}

Para determinar a capacidade de carga das estacas, foi utilizada a fórmula dinâmica atribuída a Weisbach (JUMIKIS, 1971):

$$
Q_{d}=\frac{2 \cdot e_{H} \cdot W \cdot h}{s+\sqrt{s^{2}+2 \cdot e_{H} \cdot W \cdot h \cdot \frac{L}{E \cdot A}}}
$$

onde $Q_{d}$ é a resistência (dinâmica) mobilizada ao final da cravação, $e_{H}$ é a eficiência do martelo de cravação, $W$ é o peso do martelo, $h$ é a altura de queda do martelo, $s$ é a nega, $L$ é o comprimento da estaca, $E$ é o módulo de elasticidade do material que compõe a estaca, e $A$ é a área da seção transversal da estaca.

Para este trabalho, a fórmula original de Weisbach sofreu uma alteração, substituindo-se o comprimento real $L$ pelo comprimento equivalente $L^{*}$. O comprimento equivalente representa $\mathrm{o}$ encurtamento elástico da estaca, levando em conta a transferência de carga por atrito ao longo do seu comprimento. De uma forma geral, pode-se representar o comprimento equivalente como:

$$
L^{*}=\alpha \cdot L
$$

sendo $\alpha$ um fator de proporcionalidade, função do mecanismo de transferência de carga da estaca. Este valor pode variar entre 0,5 (estaca sem transferência de carga pela ponta) até 1,0 (estaca sem transferência de carga por atrito).

Além disso, a transformação da resistência dinâmica ao final da cravação $\left(Q_{d}\right)$ em uma capacidade de carga estática de longo prazo $\left(Q_{u}\right)$ é realizada através de um fator de correção. Este fator de correção é entendido como sendo a razão entre um fator de crescimento da capacidade de carga com o tempo ("set-up"), $F T$, e um fator de amplificação dinâmica $F D$, que representa a relação entre as resistências mobilizadas na estaca por uma ação dinâmica e por uma ação estática.

Assim, a fórmula modificada de Weisbach fica:

$$
Q_{u}=\frac{2 \cdot e_{H} \cdot W \cdot h}{s+\sqrt{s^{2}+2 \cdot e_{H} \cdot W \cdot h \cdot \frac{\alpha \cdot L}{E \cdot A}}} \cdot \frac{F T}{F D}
$$

As variáveis consideradas determinísticas na análise foram o peso do martelo $(W)$, a área da estaca $(A)$, o módulo de elasticidade do material da estaca $(E)$, o fator $\alpha$ (estimado com base em resultados de ensaios de carregamento dinâmico realizados em estacas da obra), a altura de queda $(h)$, a eficiência do martelo $\left(e_{H}\right)$ e o comprimento da estaca $(L)$. Na Tabela 1 são demonstrados os valores adotados: 
Tabela 1: Variáveis determinísticas adotadas para fórmula dinâmica adaptada

\begin{tabular}{cc}
\hline Variável & Valor \\
\hline $\mathrm{W}(\mathrm{kN})$ & 42,27 \\
$\mathrm{~A}\left(\mathrm{~m}^{2}\right)$ & 0,021 \\
$\mathrm{E}(\mathrm{GPa})$ & 210 \\
$\alpha$ & 0,5675 \\
$\mathrm{~h}(\mathrm{~m})$ & 2 \\
$\mathrm{e}_{\mathrm{H}}$ & 0,7 \\
$\mathrm{~L}(\mathrm{~m})$ & 45,129 \\
\hline
\end{tabular}

Para aplicar o Método das Estimativas Pontuais, foram consideradas como aleatórias as seguintes variáveis: a nega das estacas (s) e os fatores de correção $F T$ e $F D$. Para a nega, foram adotados a média e o desvio padrão medidos durante a obra, nas estacas da área de influência S4A. Para os fatores de correção $F T$ e $F D$, o valor médio foi obtido pela média aritmética entre os valores extremos concebíveis (máximo e mínimo). Para $F D$, essa faixa foi estimada entre 1,5 e 2,0; para $F T$, entre 2,0 e 3,0. Já o desvio padrão de $F T$ e $F D$ foi estimado a partir da faixa de variação concebível (subtração entre o valor máximo e o valor mínimo). Adotou-se a "regra dos 3- $\sigma$ ", baseada no fato de que $99,73 \%$ de todos os valores de uma variável aleatória normalmente distribuída recaem ao redor de três desvios padrão do valor médio. Porém, são reportadas na literatura situações em que a divisão da faixa de variação por 6 conduz a subestimativas do desvio padrão. Assim, no presente estudo, adotou-se a divisão da faixa de variação por 4 , conforme sugestão de Duncan (2001). Na Tabela 2 são mostrados os valores da média e do desvio padrão para cada variável aleatória.

Tabela 2: Variáveis aleatórias adotadas para fórmula dinâmica adaptada

\begin{tabular}{ccc}
\hline Variável & Média & $\begin{array}{c}\text { Desvio } \\
\text { padrão }\end{array}$ \\
\hline FT & 2,5 & 0,25 \\
\hline FD & 1,75 & 0,125 \\
\hline s (m) & 0,001 & 0,00011212 \\
\hline
\end{tabular}

Aplicando o Método das Estimativas Pontuais para uma função de três variáveis aleatórias $(F T, F D$, $s)$ considerando os coeficientes de correlação nulos entre elas, foram obtidos para a média $(\bar{C})$, o desvio padrão $\left(\sigma_{C}\right)$ e o coeficiente de variação $\left(\delta_{C}\right)$ da capacidade de carga das estacas, os resultados mostrados na Tabela 3. 
Tabela 3: Resultados obtidos na análise da capacidade de carga das estacas

\begin{tabular}{cc}
\hline Parâmetro & Valor \\
\hline $\bar{C}(k N)$ & 6239,8 \\
\hline$\sigma_{C}(k N)$ & 768,6 \\
\hline$\delta_{C}$ & $12 \%$ \\
\hline
\end{tabular}

\section{ANÁLISE PROBABILÍSTICA DAS CARGAS SOBRE AS ESTACAS}

De acordo com o memorial de cálculo estrutural, as estacas da obra receberão em seu topo uma carga característica de compressão $\left(F_{k}\right)$ igual a $3000 \mathrm{kN}$, oriunda das diversas combinações possíveis de cenários de carregamento sobre o cais. As cargas características $F_{k}$ podem ser representadas pela soma de duas parcelas:

$F_{k}=G_{k}+Q_{k}$

sendo $G_{k}$ a parcela permanente das cargas aplicadas à estaca e $Q_{k}$ a parcela variável das cargas aplicadas à estaca.

No presente estudo, aplicou-se o Método das Estimativas Pontuais para uma função de duas variáveis aleatórias, considerando como variáveis aleatórias as cargas permanentes $\left(G_{k}\right)$ e as cargas variáveis $\left(Q_{k}\right)$. Neste procedimento foram adotadas três análises, para três valores de $r$ distintos, onde $r$ representa a relação entre as parcelas permanente e variável da carga característica:

$r=\frac{G_{k}}{G_{k}+Q_{k}}=\frac{G_{k}}{F_{k}}$

A razão $r$ varia de acordo com o tipo de obra. Foram adotados neste estudo valores de $r$ iguais a 0,5 , 0,7 e 0,9 . O coeficiente de correlação entre as parcelas permanente e variável $\left(\rho_{G Q}\right)$ foi considerado igual a 0 ou 1 . Os resultados referentes à média $(\bar{D})$, o desvio padrão $\left(\sigma_{D}\right)$ e o coeficiente de variação $\left(\delta_{D}\right)$ das cargas características sobre as estacas estão descritos nas Tabelas 4,5 e 6.

Tabela 4: Resultados da análise probabilística das cargas adotando $r=0,5$

\begin{tabular}{ccc}
\hline Parâmetro & $\boldsymbol{\rho}_{\mathbf{G Q}}=\mathbf{0}$ & $\boldsymbol{\rho}_{\mathbf{G Q}}=\mathbf{1}$ \\
\hline $\bar{D}$ & 3000,0 & 3000,0 \\
$\sigma_{D}$ & 403,9 & 525,0 \\
$\delta_{D}$ & $13 \%$ & $18 \%$ \\
\hline
\end{tabular}


Tabela 5: Resultados da análise probabilística das cargas adotando $r=0,7$

\begin{tabular}{ccc}
\hline Parâmetro & $\boldsymbol{\rho}_{\mathbf{G Q}}=\mathbf{0}$ & $\boldsymbol{\rho}_{\mathbf{G Q}}=\mathbf{1}$ \\
\hline $\bar{D}$ & 3000,0 & 3000 \\
$\sigma_{D}$ & 307,8 & 435,0 \\
$\delta_{D}$ & $10 \%$ & $15 \%$ \\
\hline
\end{tabular}

Tabela 6: Resultados da análise probabilística das cargas adotando $r=0,9$

\begin{tabular}{ccc}
\hline Parâmetro & $\boldsymbol{\rho}_{\mathbf{G Q}}=\mathbf{0}$ & $\boldsymbol{\rho}_{\mathbf{G Q}}=\mathbf{1}$ \\
\hline $\bar{D}$ & 3000,0 & 3000 \\
$\sigma_{D}$ & 280,2 & 345,0 \\
$\delta_{D}$ & $9 \%$ & $12 \%$ \\
\hline
\end{tabular}

\section{ANÁLISE DE CONFIABILIDADE}

O índice de confiabilidade $\beta$ é o parâmetro que representa a probabilidade de falha de um elemento estrutural ou de fundação. Para este estudo foi calculado o índice de confiabilidade a partir dos resultados da análise probabilística da carga sobre as estacas e de sua capacidade de carga, através da seguinte equação que pode ser encontrada em Harr (1987):

$\beta=\frac{\bar{C}-\bar{D}}{\sqrt{\sigma_{C}^{2}+\sigma_{D}^{2}-2 \rho_{C D} \cdot \sigma_{C} \cdot \sigma_{D}}}$

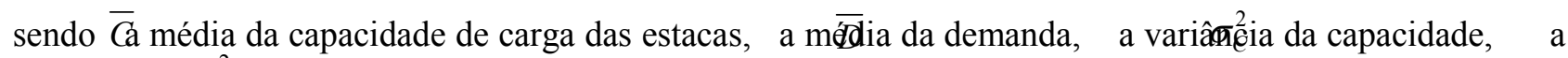

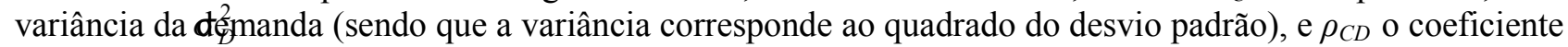
de correlação entre capacidade e demanda. Na dedução da Equação 7, as distribuições da capacidade e da demanda são consideradas normais.

Os valores para o índice de confiabilidade encontrados no presente estudo estão expostos nas Tabelas 7, 8 e 9, para diferentes valores de $r$ e $\rho_{G Q}$. Apesar de Harr (1987) afirmar que o coeficiente de correlação entre capacidade e demanda $\left(\rho_{C D}\right)$ deve ficar em torno de 0,75 , neste trabalho o valor de $\rho_{C D}$ foi considerado igual a 0 , o que reduz o índice de confiabilidade $\beta$. 
Tabela 7: Resultados do índice de confiabilidade para $r$ igual a 0,5

\begin{tabular}{ccc}
\hline Parâmetro & $\boldsymbol{\rho}_{\mathbf{G Q}}=\mathbf{0}$ & $\boldsymbol{\rho}_{\mathbf{G Q}}=\mathbf{1}$ \\
\hline $\bar{C}$ & 6239,79 & 6239,79 \\
$\bar{D}$ & 3000,00 & 3000,00 \\
$\sigma_{\mathrm{C}}$ & 768,57 & 768,57 \\
$\sigma_{\mathrm{D}}$ & 403,89 & 525,00 \\
$\rho_{\mathrm{CD}}$ & 0,00 & 0,00 \\
$\boldsymbol{\beta}$ & $\mathbf{3 , 7 3}$ & $\mathbf{3 , 4 8}$ \\
\hline
\end{tabular}

Tabela 8: Resultados do índice de confiabilidade para $r$ igual a 0,7

\begin{tabular}{ccc}
\hline Parâmetro & $\boldsymbol{\rho}_{\mathbf{G Q}}=\mathbf{0}$ & $\boldsymbol{\rho}_{\mathbf{G Q}}=\mathbf{1}$ \\
\hline $\bar{C}$ & 6239,79 & 6239,79 \\
$\bar{D}$ & 3000,00 & 3000,00 \\
$\sigma_{\mathrm{C}}$ & 768,57 & 768,57 \\
$\sigma_{\mathrm{D}}$ & 307,77 & 435,00 \\
$\rho_{\mathrm{CD}}$ & 0,00 & 0,00 \\
$\boldsymbol{\beta}$ & $\mathbf{3 , 9 1}$ & $\mathbf{3 , 6 7}$ \\
\hline
\end{tabular}

Tabela 9: Resultados do índice de confiabilidade para $r$ igual a 0,9

\begin{tabular}{ccc}
\hline Parâmetro & $\boldsymbol{\rho}_{\mathrm{GQ}}=\mathbf{0}$ & $\boldsymbol{\rho}_{\mathrm{GQ}}=\mathbf{1}$ \\
\hline $\bar{C}$ & 6239,79 & 6239,79 \\
$\bar{D}$ & 3000,00 & 3000,00 \\
$\sigma_{\mathrm{C}}$ & 768,57 & 768,57 \\
$\sigma_{\mathrm{D}}$ & 280,22 & 345,00 \\
$\rho_{\mathrm{CD}}$ & 0,00 & 0,00 \\
$\boldsymbol{\beta}$ & $\mathbf{3 , 9 6}$ & $\mathbf{3 , 8 5}$ \\
\hline
\end{tabular}


O gráfico da Fig. 4 resume os resultados alcançados no estudo.

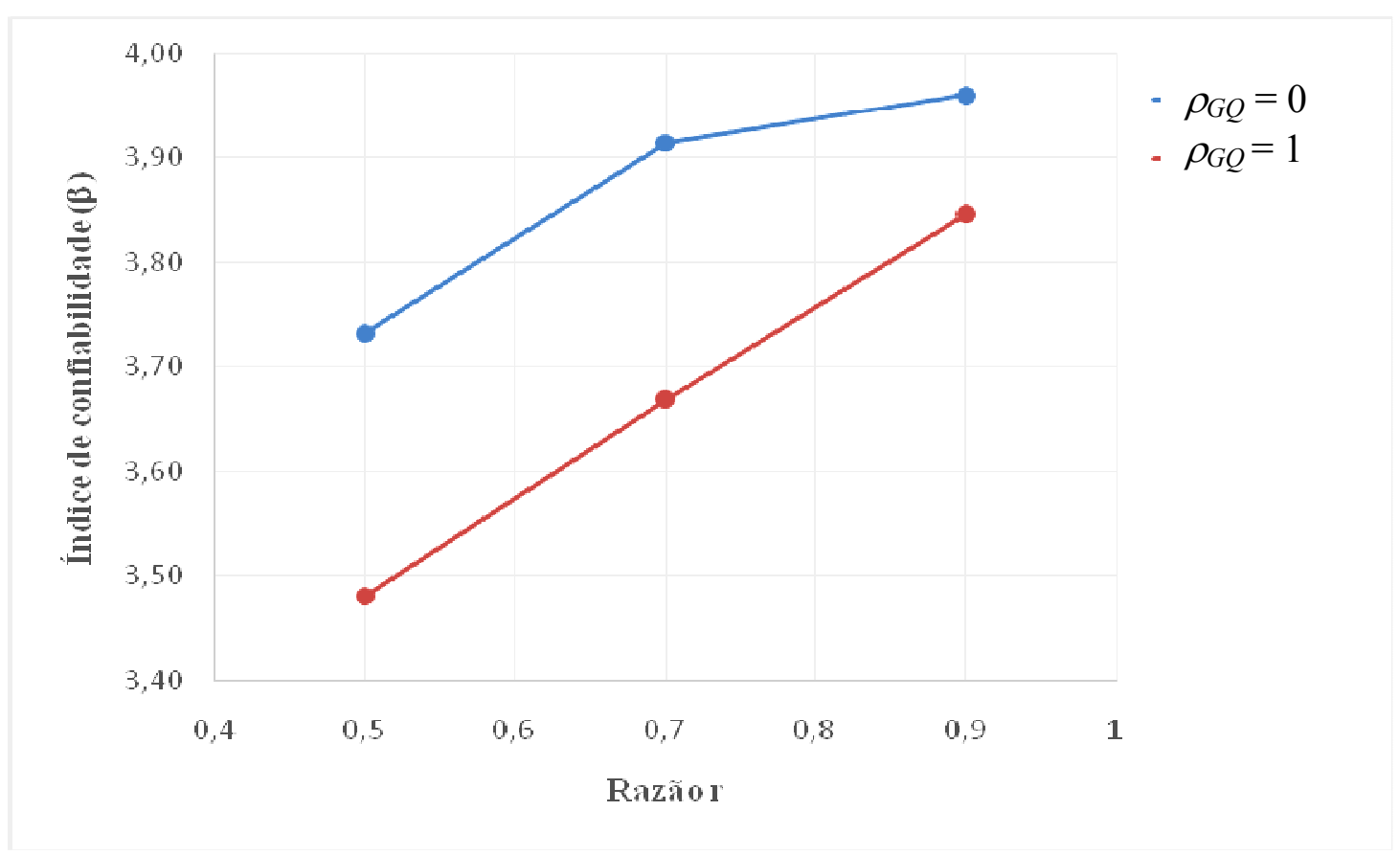

Figura 4: Índice de confiabilidade $\beta$ versus razão $r$

Pode-se observar que, conforme aumenta a razão $r$, aumenta o índice de confiabilidade $\beta$, qualquer que seja o coeficiente de correlação $\rho_{G Q}$. Também nota-se que, atribuindo-se um coeficiente de correlação $\rho_{G Q}$ igual a 0 , obtêm-se maiores índices de confiabilidade do que adotando-se o valor igual a 1 .

\section{CONCLUSÃO}

$\mathrm{Na}$ área da engenharia de fundações são geralmente considerados aceitáveis probabilidades de ruína inferiores a 1/1000 eventos (AOKI, 2002). Na Tabela 10 são mostrados os valores do índice de confiabilidade $\beta$ relacionados às probabilidades de ruína $p_{f}$, admitindo que as distribuições da capacidade $\mathrm{e}$ da demanda são ambas normais. 
Tabela 10: Valores de $\beta$ em função de $p_{f}$ (Cintra e Aoki, 2010)

\begin{tabular}{cc}
\hline $\boldsymbol{p}_{\boldsymbol{f}}$ & $\boldsymbol{\beta}$ \\
\hline 0,5 & 0,000 \\
0,2 & 0,842 \\
0,1 & 1,282 \\
0,05 & 1,645 \\
0,01 & 2,326 \\
$\mathbf{0 , 0 0 1}$ & $\mathbf{3 , 0 9 0}$ \\
0,0002 & 3,540 \\
0,0001 & 3,719 \\
0,00002 & 4,107 \\
0,00001 & 4,265 \\
0,000001 & 4,768 \\
\hline
\end{tabular}

Observa-se que probabilidades de ruína inferiores a 1/1000 eventos correspondem a índices de confiabilidade superiores a 3,09. Assim, os resultados obtidos nas análises do presente estudo indicam índices de confiabilidade aceitáveis para a obra em apreço. Ressalta-se a importância da análise probabilística e de confiabilidade para uma correta avaliação da segurança de qualquer obra de engenharia.

\section{REFERÊNCIAS}

ALVES, A.M.L.; SANTA MARIA, P.E.L. Análise probabilística de problemas geotécnicos: aplicação à argila do Sarapuí. Solos e Rochas. Vol. 24, No. 1, p. 83-93, 2001.

Associação Brasileira de Normas Técnica. 2010. Projeto e Execução de Fundações. NBR 6122. Rio de Janeiro, 2010.

AOKI, N. Probabilidade de falha e carga admissível de fundação por estacas. Revista Militar de Ciência e Tecnologia. Vol. XIX, p. 48-64, 2002.

CINTRA, J. C. A.; AOKI, N. Fundações por estacas: projeto geotécnico. Editora Oficina de Textos. São Paulo, 2010.

DUNCAN, J.M. Factor of safety and reliability in geotechnical engineering. Journal of Geotechnical and Geoenvironmental Engineering (ASCE). Vol. 126, No. 4, p. 307-316, 2000.

DUNCAN, J.M. Closure on factor of safety and reliability in geotechnical engineering. Journal of Geotechnical and Geoenvironmental Engineering (ASCE). Vol. 127, No. 8, p. 717-721, 2001. 
DE MELLO, V.F.B. Reconstruindo as bases para a geotecnia prática comparativa difundindo estatísticaprobabilidades (EP) simples e convidativas para tudo. XII Congresso Brasileiro de Mecânica dos Solos e Engenharia Geotécnica. São Paulo, p. 1271-1294, 2002.

HARR, M. E. Reliability-Based Design in Civil Engineering. McGraw-Hill, New York, 1987.

JUMIKIS, A.R. Foundation Engineering. Intext Educational Publishers, New York, 1971.

LACASSE, S.; E NADIM, F. Risk and reliability in geotechnical engineering. 4th International Conference on Case Histories in Geotechnical Engineering. St. Louis, p. 1172-1192, 1998.

LOBO, B.O. Verificação de desempenho das fundações da obra de modernização de um cais de gravidade. Trabalho de Conclusão de Curso. Universidade Federal do Rio Grande, Rio Grande, RS, Brasil, 2003.

MAGALHÃES, F. C. Análise das fundações da obra de modernização do cais do Porto Novo de Rio Grande (RS) aplicando metodologia bayesiana. Dissertação de Mestrado, Programa de Pós-Graduação em Engenharia Oceânica. Universidade Federal do Rio Grande, Rio Grande, RS, Brasil, 2011.

ROSENBLUETH, E. Point estimates for probability moments. Proc. Nat. Acad. of Sci. Vol. 72, No. 10, p. 3812-3814, 1995.

ROSENBLUETH, E. Two-Point Estimates in Probabilities. Appl. Math. Modelling. Vol. 5, p. 329-335, 1981. 